\title{
E-Learning Platform and Modern Education
}

\author{
Dingyuan Zhao
}

\begin{abstract}
-in the information age, great changes have been taken place in the ways people live and work. Network information has infiltrated into all aspects of human life, which has a huge influence on the concepts and ideas of education and to a large extent affects the ways people learn things. E-Learning platform brings a brand new concept, and is a kind of network information learning mode. E-learning emphasizes on the technology to transform and guide education, which is also known as online learning or networked learning. E-learning can be regarded both as an assist learning forms to traditional education and a self-learning mode of continuing education. It can be used for traditional educational content and internal trainings for enterprises, and it is especially a very appropriate means and methods for the engineering education. E-learning provides a brand new learning mode, in which the users can learn through internet by via the terminal.
\end{abstract}

Index Terms_information; education; E-Learning; platform; modern

\section{INTRODUCTION}

As information technology and network technology developed, knowledge transfer and update very fast, people's work and life rhythm also becomes faster, in order to meet the economic development brought by the information technology, people want to have to a variety of ways of learning, E -Learning platform appeared to adapt to the needs of social development, providing people with a pan in ways of learning.

E-Learning platform is a digital media technology, emphasizing the use of technology to transform to guide education. Provided with digital resources through the network, as long as there is a network and accessing equipment, people can use resources for learning, with the features of Anyone, Anytime, Anywhere and Any-device. For different people, different customers, different needs, it can integrate and classify digital resources to form characteristics of E-Learning platform.

A new way of learning will change people's lives, but it is also need time to be accepted by all the people, which is not entirely depended on people's thinking and understanding, but depended on the usefulness, availability, and convenience that measure how E-Learning Platform can provide resources to the users. E-Learning platform is a new learning environment consists of multi-media learning resources, online learning community and network technology platform, in which it brings together a large amount of data, files, programs, educational software, interests, discussion groups, news groups and other learning resources, forming a highly comprehensive integrated resource library. A highly comprehensive integrated resource

Manuscript received June 18, 2011; revised July 6, 2011.

Dingyuan Zhao is with the School of Information Science and Technology Chengdu University (dyz1111@126.com). library in the early stages is difficult to adapt to all the needs of different customer groups, collecting and producing do not only need high-quality, high level of professional and technical personnel, but also need to integrate into an educational concept, need to constitute resources from the perspective of psychology and education, from the customer's point of view, and also a greater financial support. From the present point of view, building resource library is the major bottleneck for E-Learning platform to promote.

\section{The Composition of E-LEARNing PlatForm}

E-Learning platform consists of three major components, resource library platform, and network and access terminal. Structure is shown in Figure 1 as follow:

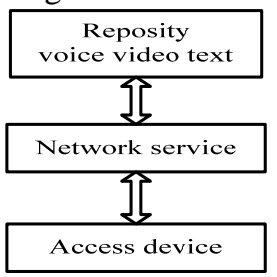

Figure 1 E-Learning structural representation

Repository platform storages a variety of audio videos and image and text information, including online resources and forms of real-time counseling, and also a discussion platform, which is the core of E-Learning; network communication using the current form; access terminal according to the user's different circumstances, can be a PC, laptop, PDA, ipad, etc.; two-way data flow indicates the E-Learning platform is interactive. E-Learning platform requires mature technology, which is used to constitute a new mode of learning, and the assurance of technology can make sure the providers focusing on the construction of the reposit

Resource library is the core of E-Learning platform, which determines the survival value and meaning of E-Learning platform. In general, resources in the library can not be produced completely by the team itself, sharing information from the information society the offers a lot for E-Learning platform, but the core resources and special resources should be produced only by the team. The composition of E-Learning platform shown in Figure 2 as follow:

We can be seen from the figure, Video Frequency data is the core resources; Real Time help is an important support resource; discussion group is an important interactive resources; and Text data, Program data, News data is an important supplementary resources, all of them integrated together form the current core part of E-Learning. At present, the core resources and support resources are the most important part, mainly for people to learn and to help them withhold. 


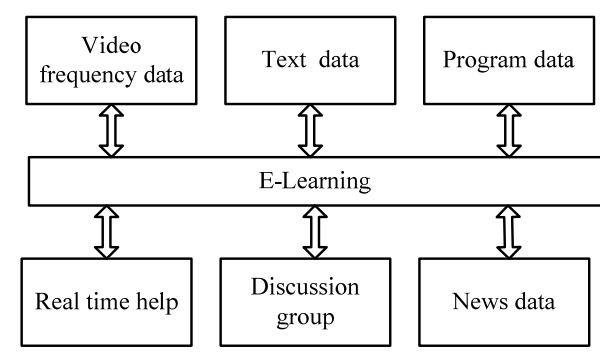

Figure2. E-earning structure

E-Learning platform provides a Web-based platform, is a whole new way for people to learn. Technically, web technology, video technology, and network communication technology, terminal access to technology is already mature. Video Frequency data acquisition and production as a core of E-Learning platform, and video resources is a open resources for public and enterprise groups. The content can meet the needs of users or not, the user through the use of E-Learning platform, can achieve the desired expectations or not? These depend not only on Video Frequency data of the video image and voice effects (these are the areas of technical staff), but more importantly, is on the content and concept provided. From an educational point of view, learning the target, defining capacity, the systematic of knowledge, practical reference guide, interests of the form, all these aspects are very important. This is not a general professional engineering and technical personnel, management personnel can fully adapt to, and not an ordinary educator can be individually competent. So video frequency data production needs to access to a team that contains all the relevant parties, team composed of personnel with the E-Learning platform related users.

In the information age, data and information in Video Frequency data repository will not be entirely its own making, using the sharing resources can bring the authorized Video Frequency data into your own E-Learning platform, but this need us pay attention to intellectual property rights. Sharing mandate resources can promote the development of E-Learning platform, so that this new learning can be more effectively promoted.

The production of video frequency data is the core part of E-Learning platform, thus under the premise of the division of the customer base, the level of the target groups of customers, the content, form and means of expression, are the problems the team must consider. Video Frequency data updating, real-time online help and files database should be considered uniformly in the design of Video Frequency data.

E-learning platform, including basic network platform (Network Infrastructure),and application platforms (Application Platform). Basic network platform provides the transmission of multimedia information flow for E-Learning, is the base of application platform, including the narrow basis of the network platform, enterprise computing and storage platforms, information systems, strategic management, information system security under Internet environment, Basic application service platform and interface technologies. Application platform is the platform implemented through a variety of ways, it is the core part of E-Learning platform. E-Learning platform design should be considered from different perspectives, mainly based on the following aspects:
1) Integration principle: E-Learning platforms must comply with the requirements of teaching, complete the various activities of the learning process in a unified system and reduce operational complexity and learning curve, simple to apply.

2) the principle of openness: the file storage format is open, used to support all the learning content and file format that is able to run online, and do not limit the requirements for curriculum development tools, only requires the tool to develop course content can be run on the Internet and users can easily access to.

3) Online learning and online help combined principle: users always want to have a proper way to get help right away when problems encountered in the learning process, so online help is a way must be considered when designing.

4) The principle of supporting multi-learning: E-Learning platform is to help and guide users to learn, learners have become the main body of the learning process, moreover they are geographically separated from learning platform, it is a fully new learning model. In this mode, most of the traditional teaching strategies are no longer applicable. Teaching support platform must be able to fully reflect these new ideas and new methods, it must be able to support exploratory learning, collaborative learning, research-based learning, which are adapt to discussion-style learning environment, E-Learning platform should provide the implementation of these Utility teaching strategies, and include various forms of information during the learning process.

E-Learning platform is an open information platform built on two-way interactive electronic information communication applications; platform design is based on the computer network and software and database technology, with two-way interactive features and advantages. It should take full advantage of technology in design, use appropriate means in order to provide users with convenience, which can provide the conditions for the operation and maintenance.

\section{The COMBINATION OF E-LEARNING PLATFORM AND TRADITIONAL EDUCATION MODE}

Traditional education mode contains of classroom teaching, after-school assignments, and students' after-school homework. This mode is deficient of learning atmosphere, and also lack of a real-time help. Students can not discuss widely and comprehensively in this mode, and when they encounter with experiment-related sectors, there is no effective means provided to be of assistance. By using E-learning platform, we can set the SNS discussion area, by which students can discuss online, ask questions which may be answered later, or it can arrange online help. The scope of the discussion can be far and wide. Meanwhile the discussing subject could be expanded. What's more, students can check through the internet on the issues during the process to get the right guidance, and to get the right answer impressively. For the problems that have experimental content, we can set the relevant scenarios, simulate experiment condition, set up virtual instruments, and provide experimental guidance in the form of online 
help for the related experimental procedures. And evaluation system can be set to real-time evaluate the experimental results for the results of learning, which can improve the learning interest and initiative. The character of E-learning is that it won't be limited by time, location, number of constraints, and it is flexible. It combines the traditional education mode with modern information technologies, with free time, free space, and free location restrictions, and it can form a learning environment and a new learning atmosphere once there are learning resources provided by internet, networking, and internet access devices. It also provides a mobile, flexible and effective assisted learning model, which combined with traditional teaching mode can be a great help on student' learning. The structure of the learning system can be shown in Figure 3:

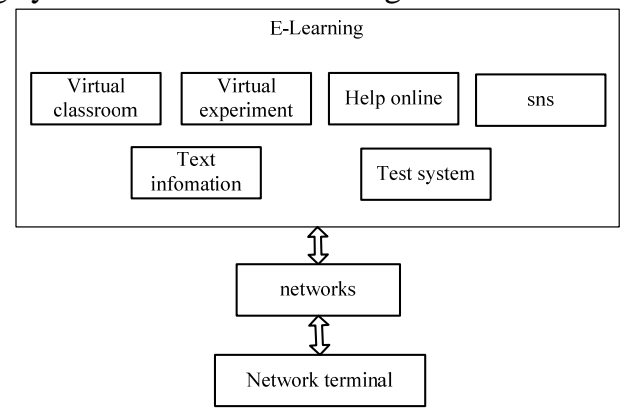

Figure 3 E-learning Assisted learning system

The figure 3 shows, Virtual Classroom can be used for students with a better and more profound understanding at the unclear points of teachers' interpretation in classroom by repeat viewing after class. It is free with the time, place, and site constraints, the experiments can be operate independently time after time, which has a promoting effect on increasing the students' interests and improving the operation ability. Students can discuss in SNS community during the learning process, which won't be affected by restrictions of time, space, and number of students. Each person can make a discussion and they can express their views on relevant issues, the issues discussed and the corresponding answers can be sort to be text information online, which is not available in the traditional class. To boot, there can be some certain full-time counseling staffs arranged to answer questions and guide the discussing issues at the same time, they can help students to establish the correct way of thinking and encourage students to creative thinking. Network information can store huge amounts of data, and by this characteristic we can store a large number of text messages on E-learning platform, which is convenient for students to query, meanwhile improve their reading abilities. We can also design online test system, through which students can test their learning results. With the network technology and information technology developed, the access mode of E-learning will become easier, which provides a good condition for E-learning application.

E-learning platform for internal training of enterprise In market economy era under the premise of information, the quality of employees has played a significant role in promoting the development of enterprise, enhancing the professional skills and the project literacy of employees is one of the effective factors to improve the enterprises' core competitiveness. Establishing managing concepts and corporate culture is an important prerequisite to maintain the development of enterprises, which also need a platform suitable for enterprise characteristics. Employees' training is long-term and continuous; and the contents update along with the change of application and developing trends of technology, meanwhile the managing concepts and corporate culture will have different characteristics at different stages. Using traditional model in the enterprise staff training is generally a short-term training, with the implementers of business leaders and some outside experts in addition. The employees have two ways of trainings, full-time training and in-service training. The traditional way is limited by time, and with single form, is very difficult to receive the desired results.

Taking advantage of E-learning's features to customized form the internal training system can provide effective internal training methods forms for enterprises.

Web-based E-learning platform can store huge amounts of data, such as audio, video information, text information, and the setting of curriculum learning, and it can plan the content and mode of assessment tests and provide online help. Internal training system based on E-learning, can classify and set the training according to actual needs of enterprises, it has a flexible learning time, and can set exam questions and forms due to specific content under the learning requirements set by the system. The structure shown in Figure 4:

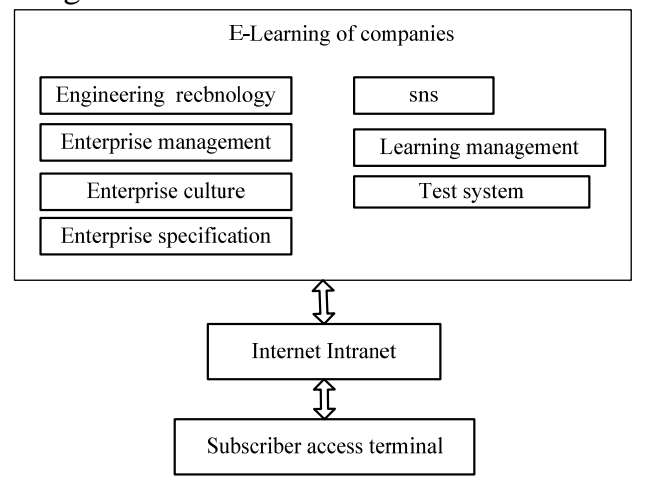

Figure 4 structure of the Corporate Training System

The figure shows, E-learning platform used for Corporate Training includes engineering, enterprise management, corporate culture and standards and norms in the enterprise, and it can combine the training contents due to needs of different roles of enterprises. When the training content was determined, the training process can be managed by learning managing system, the frequencies to go into the system and the learning time can be recorded to assess the learning process. Test system can arrange for the exams automatically due to the combined training content, mainly for objective questions currently. And if combined with manual operation, it can also put competent test and objective test together. Enterprises can just propose their own requests and outsource these functions to professional E-learning operators to lower the training costs on the basis of improving efficiency, which is also one of the characteristics of modern network platforms.

\section{E-LEARNING PlatForMS AND ENGINEERING EDUCATION}

From the British industrial revolution, engineering talents 
have always been playing a huge role in pushing forward the development of economy; countries with best engineering talents occupy the core position in economic competition and industry advantages. From the 90 s of last century, to response to issues like the shortage of engineering talent and the situation that the quality of engineering education can not meet the needs of industry, many countries set off a wave of engineering education reform. The engineering education based on CDIO (Conceive, Design, Implement, Operate), inheritance and improve the concept of engineering education reformation in Europe and the U.S. in recent 20 years, more importantly, it also proposed the capacity-building of system. Taking advantage of E-learning platform combined with the concept of CDIO, we can build an engineering practice teaching platform in some engineering professionals.

Software engineering is one of the engineering professionals which are suitable for implementing engineering education by E-learning platform, software engineering is very practical, in addition to required skills in programming, it also need to grasp - manage and control the entire software project. in the process of Software Engineering Teaching, we can use E-learning platform to strengthen the skills and techniques, we can also guide and counsel the practice through E-learning, and use Help on Line to achieve online $\mathrm{Q} \& \mathrm{~A}$. By collaborative development platform, the engineering concept of project process can be strengthened in the view of project organization and project management, and with standardized process steps, the completed work can be evaluated. For the Engineering skills, we can strengthen technical points time after time by using short video tutorials, and solve difficulties through online help, expanding our horizons by SNS discussion, thus understand trends of technology application and new technologies applications. Software development project is a process of teamwork, the quality control of software projects is a concrete implementation of engineering concepts, through collaborative development environment of E-learning platform we can build an engineering teaching environment for the practice of software development projects in related courses of software engineering, and improve engineering quality training of engineering talents. The structure of E-learning platform based on Software Engineering Education is shown in Figure 5:

From figure 5 we can see, audio and video resources are provided according to the users' needs, and online help can provide real-time guidance, SNS provides users with discuss community, which has the difficult on the collaborative development platform.

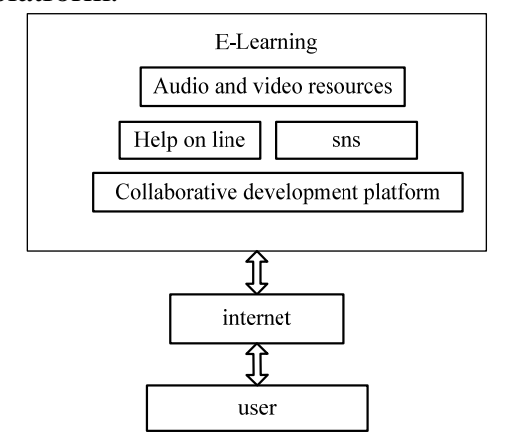

Figure 5 E-learning for Software Engineering

\section{DEVElop TRENDS OF E-LEARNING}

Although Web-based E-learning platform have outstanding features, it is unable to replace the current mode and means of education, it can only be an important aids for modern education. That is because E-learning platform can only help and evaluate objectively for the learners, without analyses human beings from areas like psychological and physiological characteristics in a subjective point of view.

1) The features of E-learning application are:

Knowledge Networking: Knowledge studying is no longer from a book, or a couple of books, but a relevant professional knowledge databases. Supported by database, the knowledge system will be re-divided, and the contents will be re -combined, learning and research methods will also have new changes.

2) Arbitrary learning: for the features that the number of employees is large and the staffs are busier than ever, E-learning is an effective solution which can meet their learning schedule needs. For the employees, the study is long-term, whether in the office, home or hotel room, as long as you can have time you can learn, thus time has become a key factor in learning. By the use of E-learning, employees can arrange study according to their schedule, not just stand to the schedule from training institutions, and this increases the degrees of learning freedom.

3) learning content update in time and continuously: From the structure and operation mode of E-learning we can see that the resources within the repository can be updated together in real-time, a variety of learning resources including learning materials maintain sync with the latest information, and the understanding of development and application trend of engineering related technology will not as delayed as the traditional education, thus the resources platform is more valuable for the staffs.

4) The immediacy of the training: traditional training staffs need to tailor for training materials, arrange training venues, and organize examination, logistics, and after that prepare for the next training. E-learning can shorten the cycle time almost, and work in real-time mode. In the days that work pace increases faster, the required learning time has exceeded the time personal and business can spend. We must use more advanced technology and design of teaching and information. If we want to keep up with the updating of knowledge.

With the above characteristics of E-learning we can see, the system still lacks a self-organized and self-learning features currently. From the perspective of the knowledge base this is a stationary accumulation and update of knowledge, and it depends on the form and content of knowledge which determined by managers. As the development of Socio-economic and scientific technology, the information is increasing, and the knowledge is more detailed classificated, there are more and more requirements for continued learning, and study is more and more clearly layered. How to gain the learning resources we need fast and effectively in a vast knowledge base is one problem to consider in the development of E-learning systems. How rich should Repository be, and how to classify and search, what features should be included in E-learning system? With the development of social economic and information technology, the designers 
should provide an intelligent E-learning system with rich resource to meet people's needs. The system stored the digital information of knowledge in a data warehouse, and by data mining and intelligent search algorithms, it receives the users' demands information, and sends the demand information to the system, after analyses and checking up, provides the learning resources due to the users' needs. To accomplish this function, we need a resources base contains huge information resources, and the classification and storage methods are directly related to the algorithm model. Thus when receiving requests for information from users', the information and relationship contained in it should be first analyzed, and then it can search query due to the users' needs. If it goes further, an expert system and learning system should be contained in the data warehouse, through which we can transform the resources which are not exist before into new learning resources and store them in the system, and then there will be an open E-learning systems. According to technological developments and demands currently, establishing an intelligent E-learning system is still hard, but combined the data warehousing with intelligent algorithms, E-learning system has already become a focus in the public, and it is one of the current directions of E-learning research.

E-learning-systems is a product of the development of social economic and IT technology, its development trends depend on the existing technology platform and the application effect of repository. For E-learning platform, the development of technology and application does not play a decisive role, the key part is still the resource library, which is established by education and technical experts working together. The concepts and forms of education and the classification and division of educational content are refined by education experts, while the technology is only built to be an application platform. In the development trend of E-learning system, Search algorithm will play an increasingly important role, and a large-scale E-learning system should have the development trends closely integrated with intelligent. Network access mode and the way to provide services are both factors that will affect its growing, thus the development of network information technology has a great impact on trend of E-learning

\section{CONCLUSION}

E-Learning platform brings people with a new mode of learning, in which learning is no longer a book of knowledge, a couple of books, but the relevant professional knowledge that transferred into the forms of data stored in the database. Under the support of database, the knowledge system will be re-divided, learning content realignment will occur, and learning research methods and new changes will occur too, learning content maintain timely and constantly renew.

E-Learning platform is in the development stage, the application model and adapted object are not yet determined, there are some E-Learning platform for specific user groups is also not yet in effect. E-Learning platform promotion is closely linked with the social and economic development, information technology development, otherwise cultural background and habits of thinking also has a great influence on the ways and contents of learning. However, under the premise of information society, the development and popularity of modern education and information technology combined E-Learning platform is the very trend.

\section{REFERENCES}

[1] LIU D. Design and realization of network long-distance study platform based on web Network Education 2005 (3): 47-49

[2] LIU Y. MA H. M. YE C. M. 'E-learning online learning and the construction of learning organization Industrial Technology and Economy'2006 (5): 37-39

[3] FU Z.T.YUE J.LI D.L.ZHANG X.S. Valuation of learner adoption intention of e-learning in china: A methodology based on perceived innovative attributes. New Zealand Journal of Agricultural Research'2007,50:609-615

[4] Guarino N. M asolo C. Content. based access to the web. IEEE intelligent systems. 1999. 14:70-80

John H. S. Seven key decision factors for selecting e-learning. Cogn c' 2 Work. 2008. 10:237-247

[5] ZHANG H.H. CHENG G. F. LI J.Q. Analysis on the present situation and its improvement of network learning assessment. Modern Distance Education. 2005.6: 26-29

[6] Jung I. Technology innovations and the development of distance education: Korean experience. Open Learning’ 2000.15(3): 217-231

[7] CHEN H.T.ZENG Q.F. Design and realization of open Study platform based on network. Science Square’ 2007.(9):160-162 